\title{
POSTGRADUATE DESIGN COURSES AS RESEARCH THINK TANKS FOR CULTURAL INSTITUTIONS
}

\author{
R. Paez; T. Montes \\ Master's Degree in Ephemeral Architecture and Temporary Spaces \\ Elisava Escola Superior de Disseny i Enginyeria de Barcelona \\ Universitat Pompeu Fabra \\ rpaez@aib.cat
}

\begin{abstract}
In the interest of reinforcing the relationship between academic research and professional practice, certain postgraduate design courses can serve as research think tanks for cultural institutions or projects which may not have access to inhouse research departments, either for organic reasons or as a result of limited resources.

This approach, which we have been implementing since 2006 within the academic framework provided by Elisava's Master's Degree in Ephemeral Architecture and Temporary Spaces, opens up a new scenario for collaboration between the academic and professional spheres through continuing alliances with different cultural institutions, where the school acts as an external research think tank for specific projects.
\end{abstract}

Key words: architectural design, strategic research, postgraduate design, cultural institutions 


\section{POSTGRADUATE DESIGN COURSES AS RESEARCH THINK TANKS FOR CULTURAL INSTITUTIONS}

In the interest of reinforcing the relationship between academic research and professional practice, certain postgraduate design courses can serve as research think tanks for cultural institutions or projects which may not have access to inhouse research departments, either for organic reasons or as a result of limited resources.

This approach, which we have been implementing since 2006 within the academic framework provided by Elisava's Master's Degree in Ephemeral Architecture and Temporary Spaces, opens up a new scenario for collaboration between the academic and professional spheres through continuing alliances with different cultural institutions, where the school acts as an external research think tank for specific projects. Over a period of eight years, we have implemented this collaborative approach, working in conjunction with the Sónar festival as well as a number of projects organized by Barcelona's Culture Institute (ICB), most notably the International Poetry Festival (FIPB).

The origins of this approach are rooted in two fundamental questions.

On the one hand, we believe that postgraduate degree programs are an opportunity for combining training and knowledge creation. Beyond teaching students how to perform the tasks inherent to the profession, postgraduate studies need to educate professionals to generate knowledge so they will be able contribute to the development of the discipline throughout their future professional careers. This is one of the qualities that has the most potential for adding value to a future architect's professional profile, and the university is without a doubt the ideal place for acquiring it. We believe that postgraduate programs should not be limited to simulations of conventional professional practice in terms of their teaching methodology. That type of exercise is necessarily incomplete due to the impossibility of reproducing a situation that mirrors actual practice, incorporating all of the factors that inform professional decision-making process. In order to be effective, that kind of approach needs to include professional internships in the curriculum, which takes away from the opportunity of generating knowledge in the academic sphere.

On the other hand, most cultural institutions and programs do not have in-house research departments. This is often the result of limited resources, as well as a lack of traditional precedents in the sector. Whereas in private industry, a stable dynamic of research and development with close ties to university research groups has been consolidated, in the cultural sector this type of dynamic is largely non-existent. Cultural projects dedicate most of their energy and their often limited means to organization, programming and management. More often than not, they do not have resources to allocate toward assessment, evaluation and strategic planning - factors which could contribute significantly to the development and improvement of key aspects in their projects. This lack is a need that has yet to be addressed, and it is an excellent opportunity for building bridges with the academic world in the form of strategic research projects.

Bringing these two questions together results in an ideal setting for creating a symbiotic relationship between the academic sphere and the realm of cultural 
production, where needs on both sides are satisfied reciprocally at the same time, generating a virtuous feedback cycle .

Elisava School of Design and Engineering has a long tradition of collaborating with businesses - especially in the areas of product design, communications and graphic design - through research and development projects. This activity has proven very effective in fostering knowledge creation and in consolidating the school as a think-tank which companies refer to when they are interested in hiring new professionals. Until recently, however, this collaboration had not been carried over into the cultural sphere.

The traditional collaboration model is tailored to the field of applied research and it relies on the typical working methodology of a design workshop: students develop proposals, individually or in groups, in response to a single design problem; the results are specific solutions that are geared toward a direct application, and the variety among the proposals is derived essentially from the students' individual interests and capabilities and from their different interpretations of the initial proposition.

Translating this model into the cultural sphere implies a shift in focus - from applied research to strategic research - and it requires a different working methodology. The processes of analysis, reflection and diagnosis, as well as the construction of the problem itself, are a fundamental part of the task and the proposals need to be developed along strategic lines. From our point of view, this differential trait is relevant because the change in methodology it implies has a decisive impact on students' education: the emphasis is placed on developing critical thought and strategic thinking. These skills are distinctive traits of architectural training and they are increasingly sought after in the professional world. Rather than providing a response to a pre-established demand, architects need to be able to construct the problem based on diagnosis and strategic thinking. Diagnosis is largely founded on an operative acknowledgement of the opportunities present in conditions which are often accepted acritically as a given. Strategic thinking involves developing the ability to make design decisions without being limited by the horizon of mere resolution.

Although strategic research, as we mentioned earlier, is among the specific qualities inherent in architecture and the design disciplines in general which holds the most potential, it is not often the focus of much attention in the formal curriculum.

In terms of academic methodology, developing an ability for strategic thinking implies a two-fold movement. First, during what we call the instruction phase, students are familiarized with the tools they need to outline a strategic working framework. Second, during what we call the operation phase, students put these specific tools into action: their design decisions are directed toward developing strategic proposals which incorporate multiple possibilities for implementation.

The instruction phase is fundamentally instrumental, and the work is focused on two central issues: research hypothesis and mediation systems.

The definition of the research hypotheses is done in student groups and with the close participation of professors and representatives from the cultural institutions involved in the collaboration (hereafter, the partner). In this phase, first of all, the 
partner's demands are discussed and identified; second, strategic approaches are proposed which relate the partner's interests with the general conditions of the working framework (program, location, narrative, etc.); finally, the specific working hypotheses are agreed upon, with a particular focus that is different for each working group. In the end, this process results, on the one hand, in the focused construction of the problem (what is the object of the strategic research?) and, on the other, in the definition of a specific working hypothesis for each group. In this way, each of the projects focuses on developing a specific application of the general research framework determined jointly by the client, the students and the professors. In contrast with a conventional design workshop structure - which is often focused on resolution, through the development of multiple responses to the same question- this strategic working structure is based on approaching the initial problem in different ways, so that the project is rooted in problematization as opposed to solely providing solutions. [Fig. 1].

After defining a research hypothesis, the other area in the instruction phase centers on mediation systems. We use the term mediation systems to refer to formats that establish a system for mediating between designer and design, through a protocolization of reality (on a programmatic, spatial or social level) based on a specific point of view. These abstract intermediaries are not only able to read specific aspects of reality; they inform design decisions on a strategic and tactical level, and in terms of resolution. The most well-known mediation systems used in the design disciplines are the map and the diagram, but other examples include scripts, scenarios, scores, spatial formats or protocols. They are all abstract intermediaries that operatively inform the designers' decision-making process by mediating between disparate levels of 'objective' reality and 'subjective' architectural aims. Mediation systems offer alternatives to the idea of design understood as composition by generating an intersubjective filter which allows for establishing links between the different agents in play, while activating a dialogic mediation among the different levels of objective reality and subjective desires. [Figs. 2 and 3].

The operation phase is fundamentally productive, and it follows a methodology divided into four movements: strategic interpretation of the program through diagrams; strategic interpretation of the site through maps; operations on these diagrams and maps; and the development of prototypical proposals.

Diagrams and maps are used to uncover hidden potential and to reveal strategic opportunities both in the cultural program contributed by the partner and in the physical framework where that program takes place [Figs. 4 and 5]. Beyond contributing new information regarding place and program, these operations explore potentials for intervention; in other words, they establish a specific framing for the factors which need to be negotiated in the project, and they construct an authorial position based on defining valid or relevant intervention strategies. Later, the mediation systems which have been investigated are put to work to make design decisions through operations on those maps and diagrams [Figs. 6, 7 and 8]. In this way, the active perspective on the problem that has been constructed from a specific standpoint is given a projective impulse which fuels proposals - still on a strategic level. A prescriptive phase comes last, beginning with the development of prototypical proposals that explore concrete options for implementing the strategic proposals. The work done in this phase usually provides a framework for possible versions or local variations that could ultimately be designed. In order to concentrate efforts on developing the strategic 
research and thinking consistent with our understanding of academic research, and to avoid any intrusion from postgraduate programs into the professional sphere, students' design proposals are not carried over into the realm of specific applications, i.e., providing concrete solutions which could be implemented directly by the partner. The aim is not to design professional projects, but to engage in a type of research and prospection which is usually neglected both by cultural institutions, due to a lack of resources, and by professionals, due to a lack of training. [Fig. 9].

Outside the strict academic framework there is a third phase, the application phase. It aims to ensure continuity in the collaboration between cultural and academic institutions, establishing feedback between the strategic research developed by the school and the continued applied research in the institutional context, either public or private. These joint efforts with cultural institutions are formalized through collaboration agreements. In addition to planning and preparing the aforementioned research projects, this includes a commitment to incorporating students through professional internships. The system of grants and internships at the cultural institutions we partner with is meant to ensure that, once the academic program is finished, students will be able to continue the work they began in the Master's program, ultimately transforming strategic research into applied research.

In conclusion, the goal of postgraduate courses understood as think thanks for cultural institutions is, first, to contribute to conceptualizing the demands made by the institutions we work with in order to clearly define areas of opportunity for design; second, to construct strategic research that can help bring into focus which areas are relevant in terms of design decision-making, regardless of the particularity of those decisions; and, third, suggesting prototypical solutions which, without moving beyond the strategic realm defined by this methodology, point out possible avenues for architectural intervention for the benefit of cultural institutions.

We believe this approach is effective in three specific aspects: it contributes decisively to reinforcing the link between the school and the network of institutions and companies in the cultural sphere; it contributes to reasserting the role of architecture as an eminently cultural activity; and it is a means of broadening professional horizons for future practitioners beyond traditional options. 


\section{IMAGES AND FOOTNOTES}

Unless otherwise indicated, all of the images that accompany this article were produced in the context of postgraduate programs directed by the authors at Elisava.

Further information can be found in:

Montes, Toni and Roger Paez, eds. Design Strategies for Temporary Intervention in Public Space. Barcelona: Elisava, 2014. ISBN 978-84-616-3481-1.

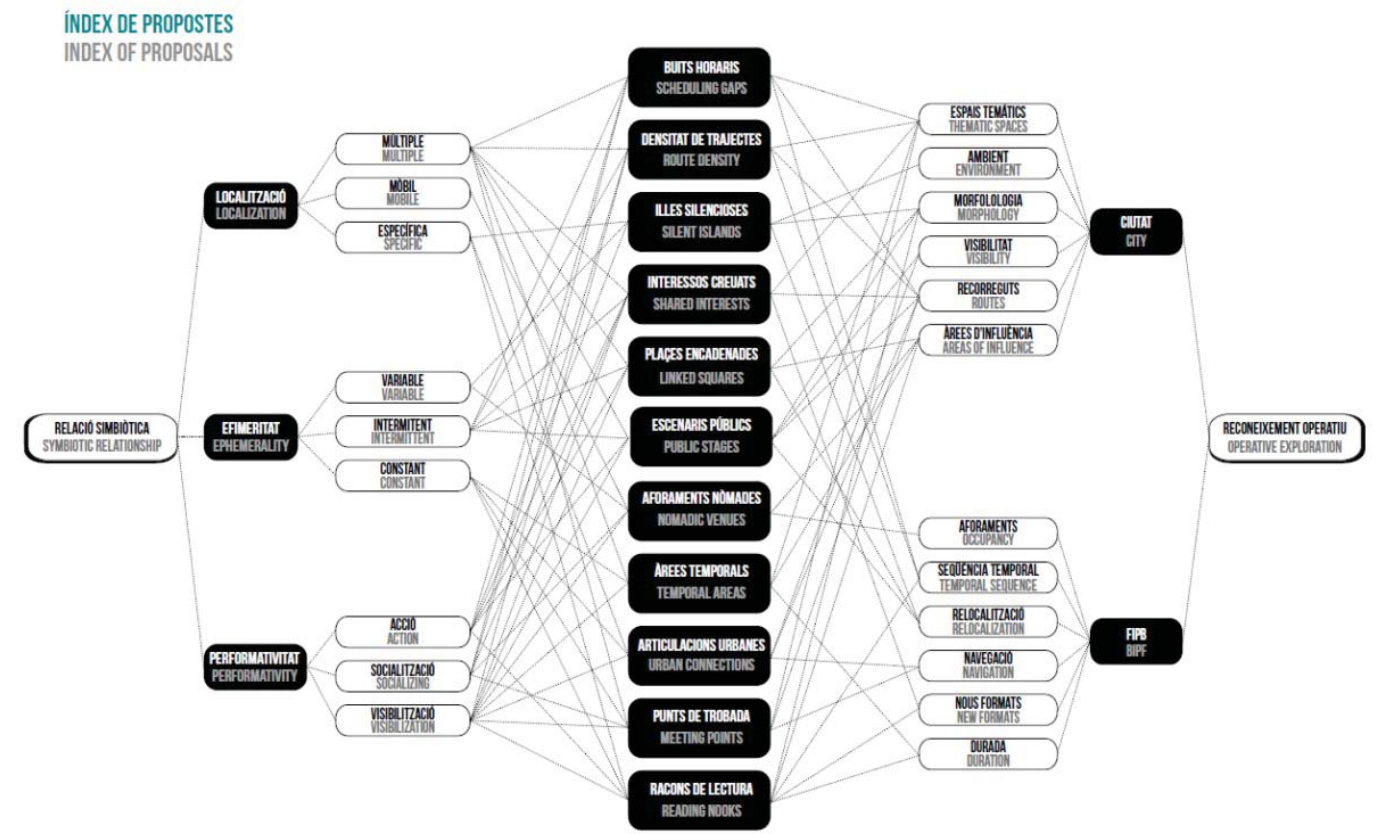

Figure 1 shows the structure for defining research hypotheses in the context of the Barcelona International Poetry Festival. Each working group structures its research through a unique combination of factors. The final result allows for a two-fold interpretation: on the one hand, it provides a set of strategic responses which refer to the case study; on the other, it provides for a prototypical illustration of a series of possible solutions corresponding to each of the strategies. 


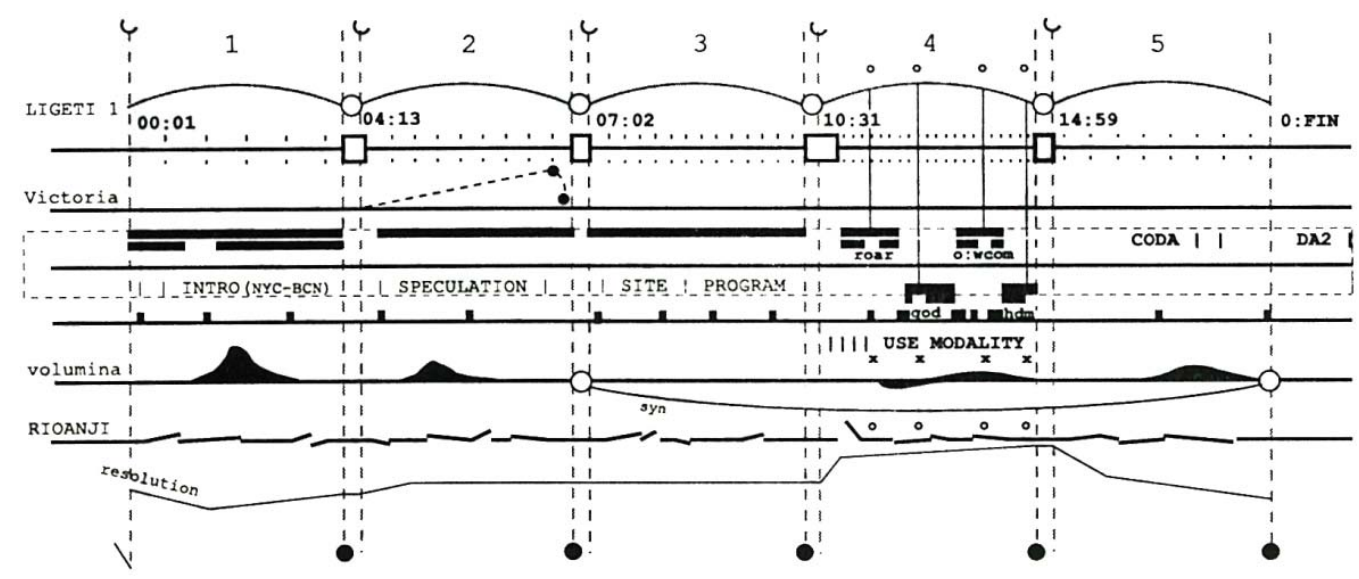

[E] : pentagrama

Figure 2 [AiB arquitectes, Roger Paez] shows a mediation system in the form of a diagram which establishes a relationship between different aspects involved in the definition of an event. Organized like a musical staff, the diagram allows for making a diachronic record of the different lines ( narrative, environmental, perceptive, etc.), while it also provides the possibility of negotiating their mutual relationships synchronically. The document is analytic and anticipatory at the same time; it is both representative and generative: while serving as a record, it also acts as a negotiation protocol for the multiple agents involved in the generation of a temporary event.

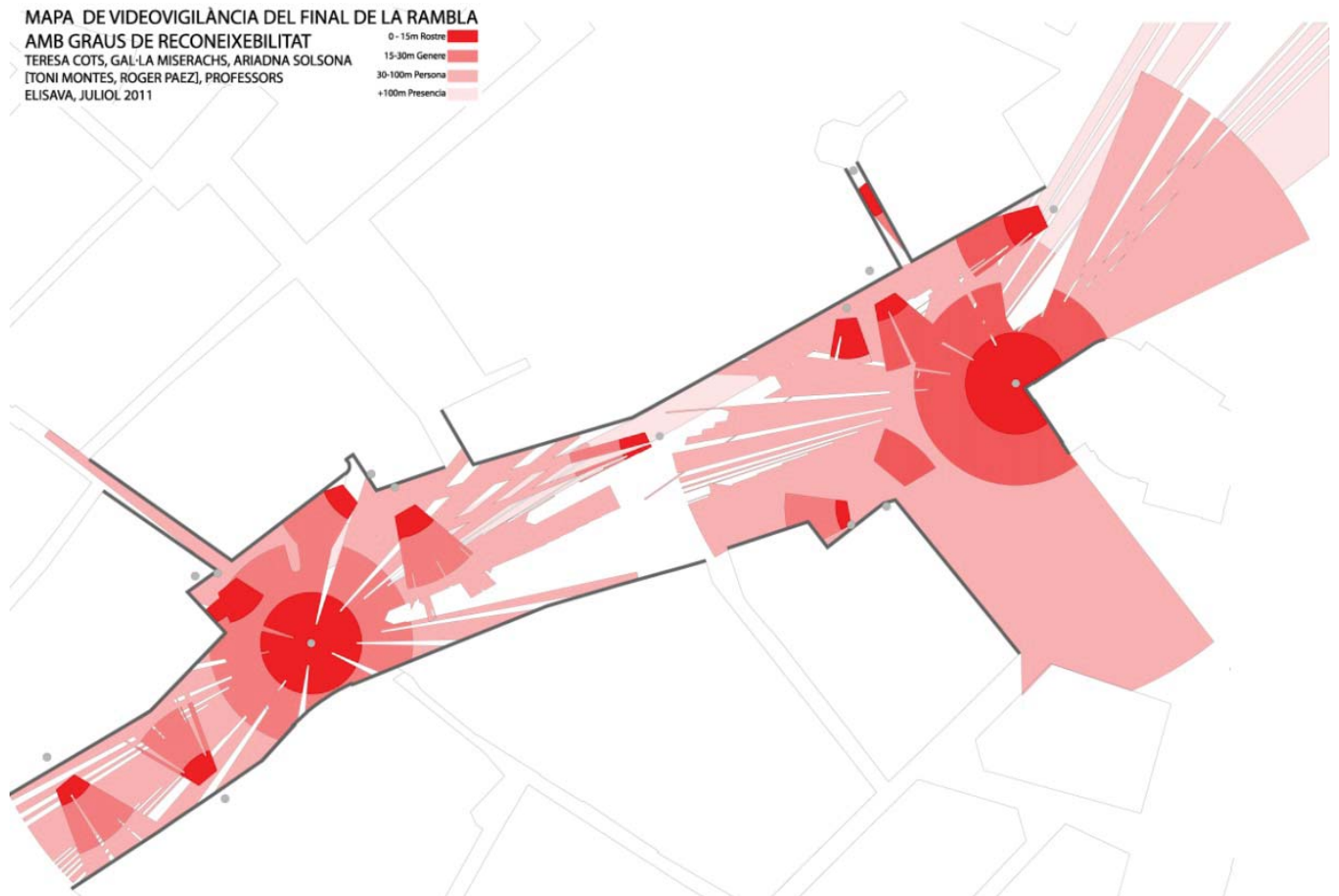

Figure 3 shows a video surveillance map for the final stretch of Barcelona's La Rambla. Following an in-depth study of the location, quantity and type of CCTV cameras, the map was created based on a systematization of the graphic code, which reveals the different degrees of surveillance in this public space. The overlapping of the different cameras' fields of vision provides a rich and nuanced reading of the levels of video surveillance, and it allows for discovering opportunities for a temporary intervention along the lines of the Surveillance Camera Players. Similarly to the previous figures, this map is both a representative and generative document: at the same time it interprets the existing reality and projects possible realities, effectively expanding the field of the real. 


\section{AFORAMENTS NÒMADES}

NOMADIC VENUES
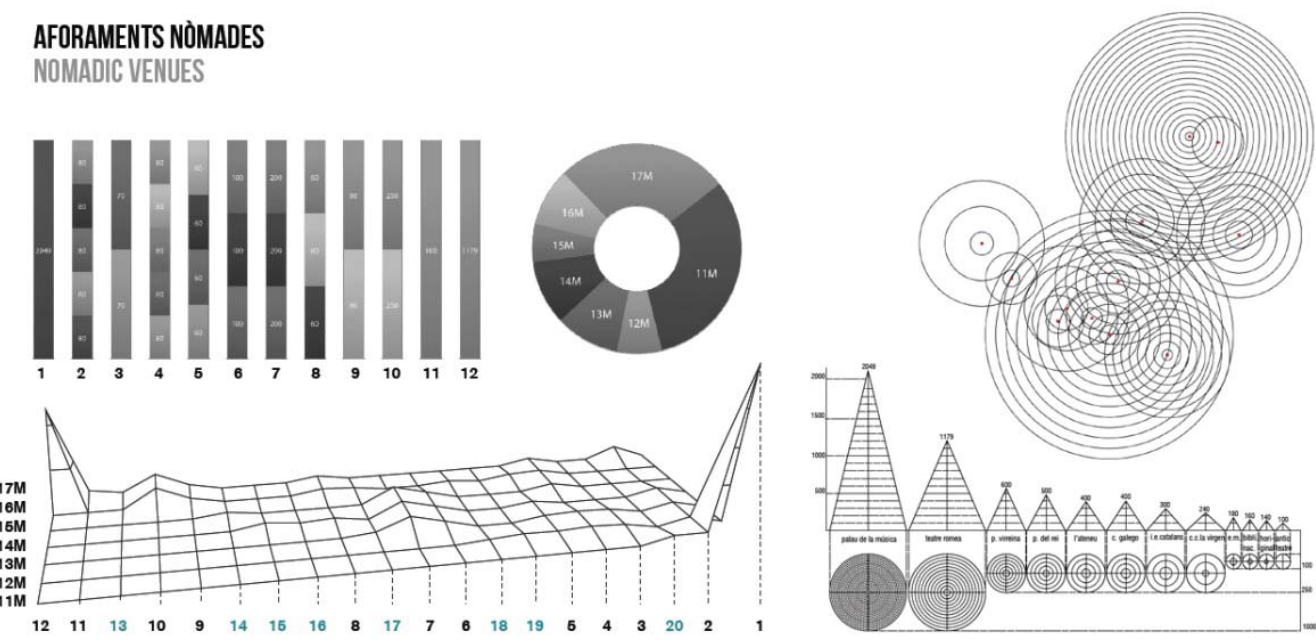

Figure 4 shows a graphic coding of numerical information, in this case the audience volumes for the Barcelona International Poetry Festival, which are highly variable from one day to the next, both in terms of audience size and according to the number of venues where events have been programmed.

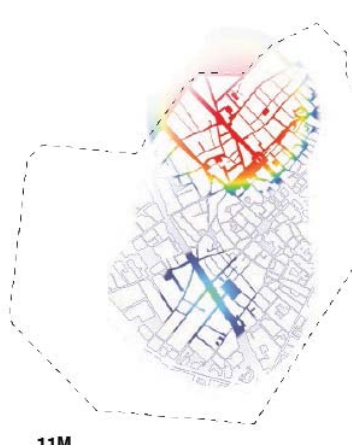

$11 \mathrm{M}$

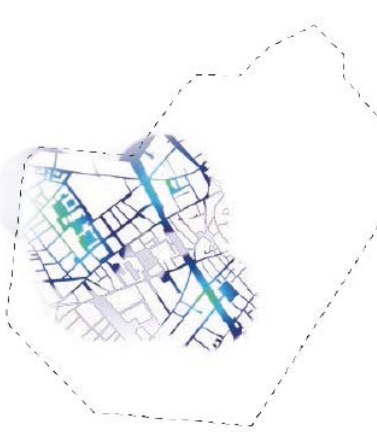

$12 \mathrm{M}$

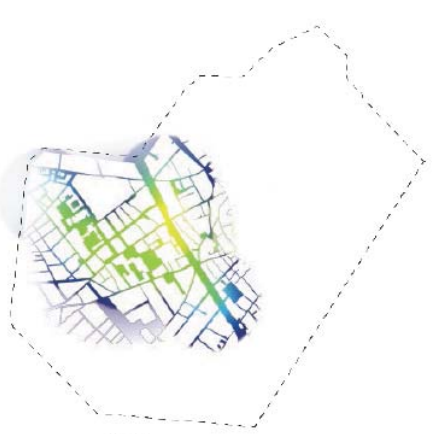

$13 \mathrm{M}$

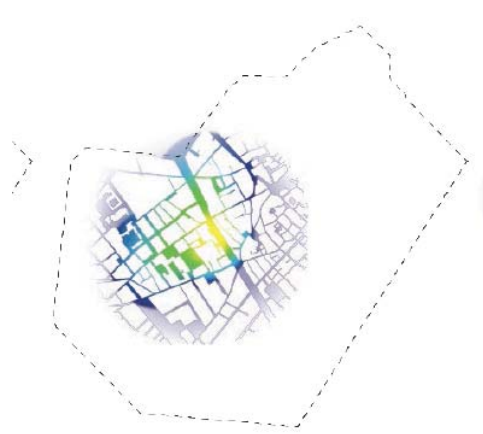

$16 \mathrm{M}$

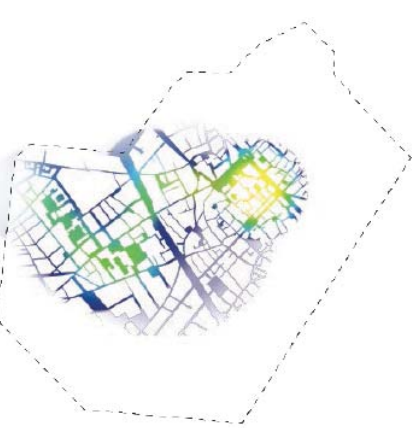

$16 \mathrm{M}$

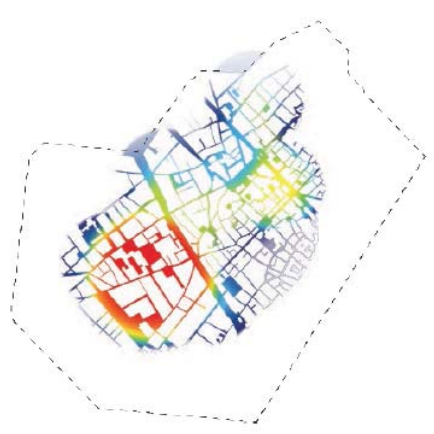

$17 \mathrm{M}$

Figure 5 shows the result of the previous coding superimposed on the map of the city, creating a dynamic map that reveals the variability of typologies and the distributions of audience hotspots over the six days of the festival's duration. 

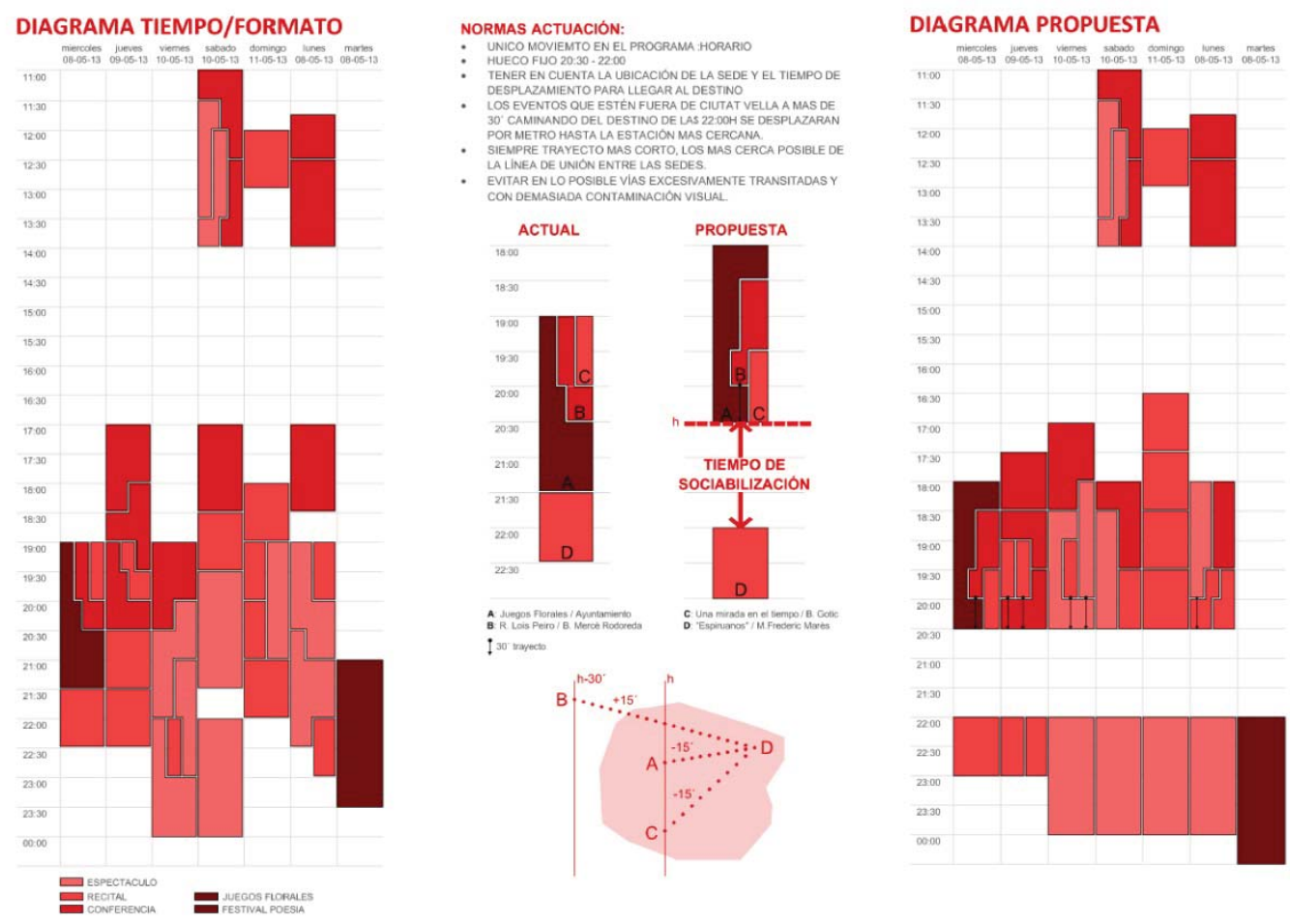

Figure 6 shows the discovery of design opportunities in the analysis of the programming for the Barcelona International Poetry Festival. First, all of the events are put onto a graph based on their scheduled times, their duration and their format. Second, the intervention criteria are defined: avoiding dissipation of the audience by promoting a common space-time for audience members to socialize before the final event for each day. Third, the maximum number of events in the afternoon are redistributed on the schedule, accounting for the travel time needed to arrive at the final event in cases where venues are far away.
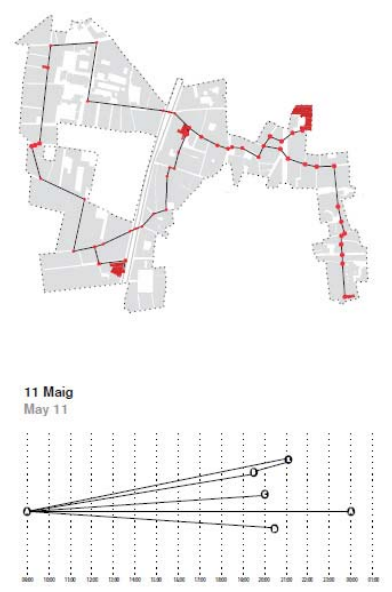

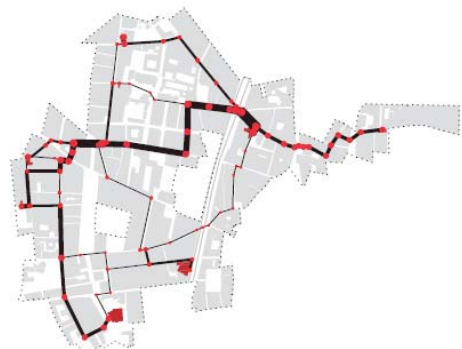

12 Maig
May 12

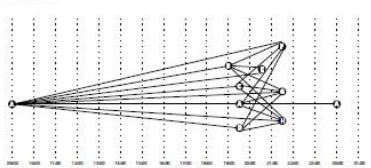

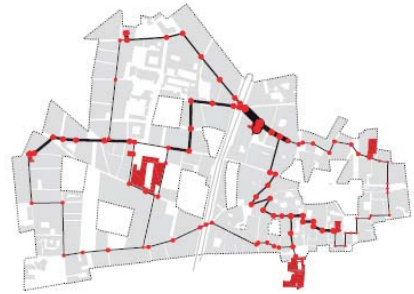

16 Maig
May 16

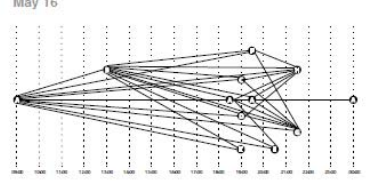

Figure 7 shows a navigation map of Barcelona's historical center based on the programming for the Barcelona International Poetry Festival. The diagrams incorporate the scheduling distribution of events for three different days, identifying all of the possible combinations for event attendance. The corresponding maps show the priority paths for travelling from one event to the next, as well as their recurrence. 


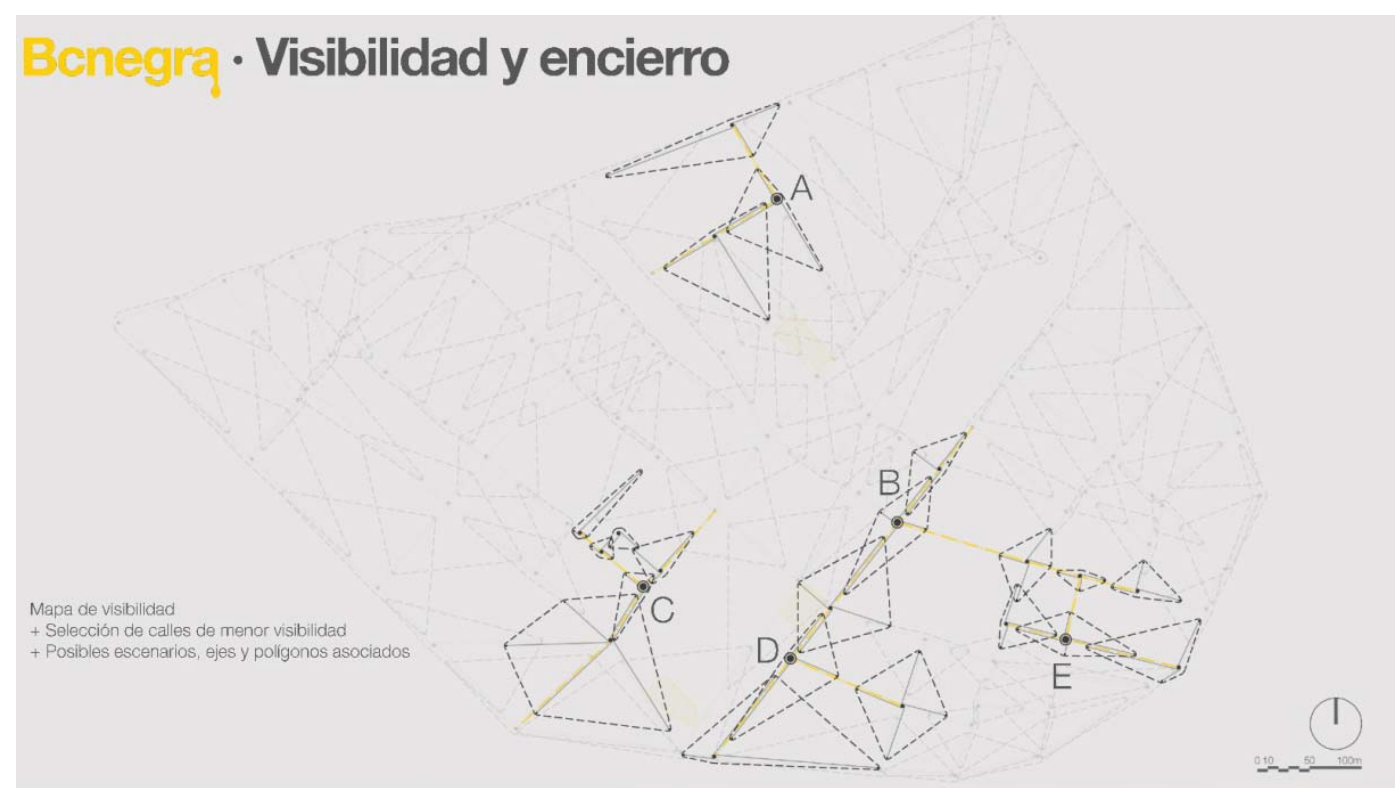

Figure 8 shows the advanced stages of an operative map generated to inform design decisions for an ephemeral intervention for Barcelona's crime fiction gathering, BCNegra - in this case, the search for the optimal location for an event to be held in public space, which requires both an area that provides good visibility and an exit route with reduced visibility. The map provides a re-reading of the urban reality based on the recognition of a limited series of parameters which are relevant to this specific project. The way in which a particular operative vision of the city is constructed is a design in and of itself.

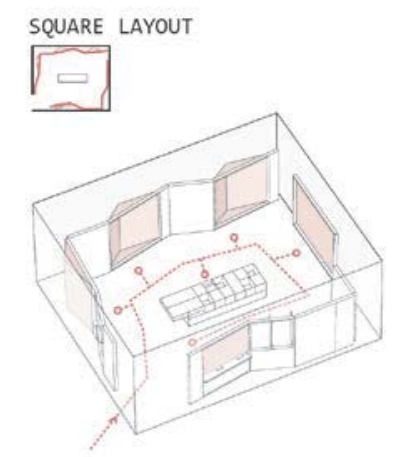

CLOSED LAYOUT

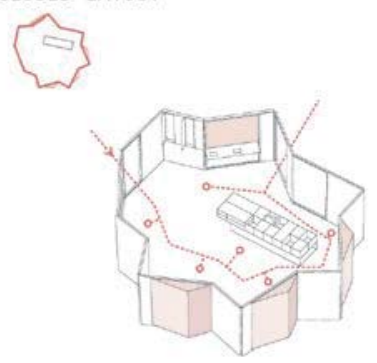

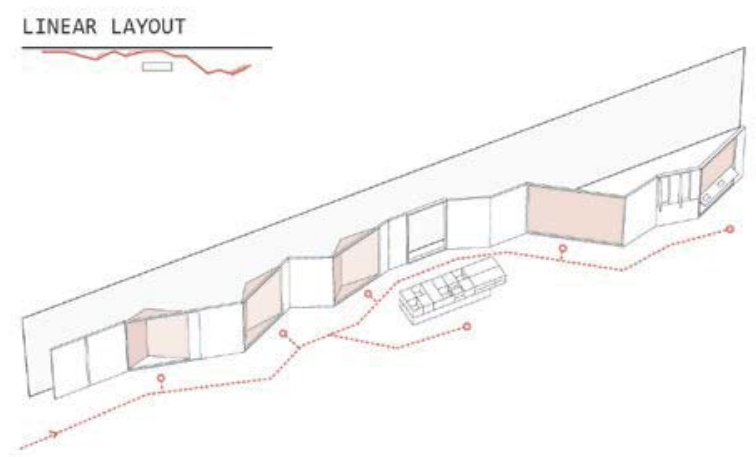

OPEN LAYOUT

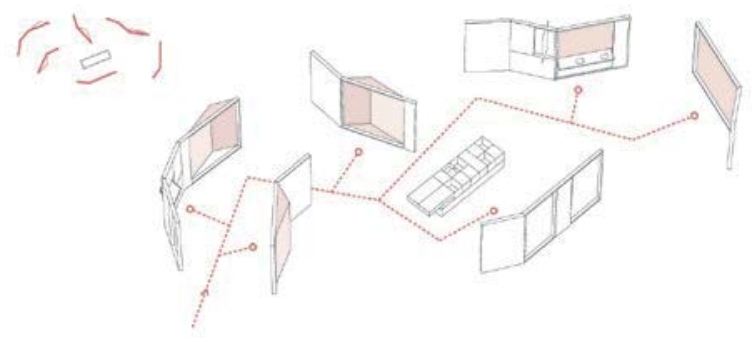

Figure 9 [miba architects, Toni Montes] shows a prototypical approach to creating a travelling exhibition mounting system, which can be disassembled and adapted to multiple spatial configurations. The system responds to open hypotheses concerning possible spaces for installing the exhibition. 